\title{
Points on elliptic curves over finite fields
}

by

\author{
M. SkaŁba (Warszawa)
}

The main result of the present paper asserts that given a polynomial $f(X)=X^{3}+A X+B \in \mathbb{Q}[X]$ with $A \neq 0$ we can find $X_{1}, X_{2}, X_{3}, U \in \mathbb{Q}$ satisfying

$$
f\left(X_{1}\right) f\left(X_{2}\right) f\left(X_{3}\right)=U^{2} .
$$

In fact our method gives a two-parameter solution, but even the problem of the existence of any solution is non-trivial.

As the first application we give a deterministic polynomial time algorithm which produces points other than the point at infinity on a given elliptic curve $E: Y^{2}=X^{3}+A X+B$ defined over a finite field $\mathbb{F}_{q}$, provided $A \neq 0$ and a certain $n \in \mathbb{F}_{q} \backslash \mathbb{F}_{q}^{2}$ is given. Remarkably, no subexponential algorithm for this problem has been known before (according to N. Koblitz's remarks on p. 129 of his book [2]). But the first recognition of the problem can be attributed to R. Schoof, who writes in his classical paper [7]:... in practice there is no problem in finding a point $P$, but I do not know how to prove that computing a point in $E\left(\mathbb{F}_{q}\right)$ is easy.

The restriction to $A \neq 0$ is essential for our method. Fortunately we can refer to Theorem 1 of [6], where an effective method for finding points on $E: Y^{2}=X^{3}+B$ is provided.

Our second application concerns patterns of quadratic residues. We will prove some result related to a conjecture of E. Lehmer and R. K. Guy. Moreover we interpret this conjecture in the language of elliptic curves and supplement another conjecture.

I am greatly indebted to Prof. A. Schinzel for his help and encouragement at every stage of writing this paper.

THEOREM 1. Let $F$ be any field of characteristic unequal to 2 or 3 and $f(X)=X^{3}+A X+B$, with $A, B \in F$ and $A \neq 0$. Put

$$
X_{j}(t)=\frac{N_{j}(t)}{D_{j}(t)} \quad \text { for } j=1,2,3,
$$

2000 Mathematics Subject Classification: 11G20, 11G05. 
where

$$
\begin{gathered}
N_{1}(t)=A^{2} t \sum_{j=0}^{4}\left(\sum_{2 a+3 b=3 j} n_{a, b}^{(1)} A^{a} B^{b}\right) t^{j}, \quad D_{1}(t)=\sum_{j=0}^{5}\left(\sum_{2 a+3 b=3 j} d_{a, b}^{(1)} A^{a} B^{b}\right) t^{j}, \\
N_{2}(t)=\sum_{j=0}^{6}\left(\sum_{2 a+3 b=3 j} n_{a, b}^{(2)} A^{a} B^{b}\right) t^{j}, \quad D_{2}(t)=144 A t \sum_{j=0}^{4}\left(\sum_{2 a+3 b=3 j} d_{a, b}^{(2)} A^{a} B^{b}\right) t^{j}, \\
N_{3}(t)=\sum_{j=0}^{15}\left(\sum_{2 a+3 b=3 j+3} n_{a, b}^{(3)} A^{a} B^{b}\right) t^{j}, \\
D_{3}(t)=A \sum_{j=0}^{5}\left(\sum_{2 a+3 b=3 j} d_{a, b}^{(31)} A^{a} B^{b}\right) t^{j} \cdot \sum_{j=0}^{10}\left(\sum_{2 a+3 b=3 j} d_{a, b}^{(32)} A^{a} B^{b}\right) t^{j},
\end{gathered}
$$

and the coefficients $n_{a, b}^{(i)}$ and $d_{a, b}^{(i)}$ are given in the tables at the end of the paper. Then

$$
f\left(X_{1}\left(t^{2}\right)\right) f\left(X_{2}\left(t^{2}\right)\right) f\left(X_{3}\left(t^{2}\right)\right)=U^{2} \quad \text { for some } U \in F(t)
$$

and $X_{1}(t), X_{2}(t), X_{3}(t)$ are not constant.

TheOREM 2. Let $\mathbb{F}_{q}$ be a finite field of $q$ elements, $\operatorname{gcd}(q, 6)=1$, and consider an elliptic curve $E: Y^{2}=X^{3}+A X+B$, where $A, B \in \mathbb{F}_{q}$ and $A \neq 0$. Let $T \subseteq \mathbb{F}_{q}$ satisfy the condition: if $t \in T$ then $-t \notin T$. Define a map $\Phi: T \rightarrow E\left(\mathbb{F}_{q}\right)$ as follows:

$\Phi(t)=\left(X_{j}\left(t^{2}\right), \sqrt{f\left(X_{j}\left(t^{2}\right)\right)}\right), \quad$ where $j=\min \left\{1 \leq i \leq 3 \mid f\left(X_{i}\left(t^{2}\right)\right) \in \mathbb{F}_{q}^{2}\right\}$, and $X_{i}(t)(i=1,2,3)$ are defined in Theorem 1 . Then $\Phi$ is well defined for at least $|T|-25$ values of $t$ and

$$
|\operatorname{Im} \Phi| \geq\left\lceil\frac{|T|-25}{26}\right\rceil .
$$

Corollary 1. Let $F$ be a finite field of characteristic unequal to 2 or 3 , $E: Y^{2}=X^{3}+A X+B$ an elliptic curve defined over $F$, where $A \neq 0$, and suppose a certain $n \in F \backslash F^{2}$ is given. Then we can compute a point $P \in E(F) \backslash\{\infty\}$ in time polynomial in $\log |F|$. The relevant $O$-constant is absolute.

Thus, according to N. Koblitz's remarks ([2, p. 129]), we can say that the main obstacle to a deterministic polynomial time algorithm for finding a point on $E$ has been overcome. The remaining obstacle, the theoretical difficulty of extracting square roots, can also be overcome in the following special case.

Corollary 2. Let $E: Y^{2}=X^{3}+A X+B$ be an elliptic curve, where $A, B \in \mathbb{Z}$ and $A \neq 0$. If $p \nmid 4 A^{3}+27 B^{2}$ then we can find a point 
$P \in E\left(\mathbb{F}_{p}\right) \backslash\{\infty\}$ in time polynomial in $\log p$. The relevant $O$-constant depends only on $|B|$.

Remark. Our method of finding points in $E\left(\mathbb{F}_{q}\right)$, based on Theorem 2, gives actually any number of points that is of polynomial growth in $\log q$, in time polynomial in $\log q$. For the sake of simplicity of formulation we have confined ourselves in the above corollaries to single points $P \neq \infty$.

There are many papers devoted to investigation of patterns of power residues. We are interested in the following special problem. Let $a<b$ be positive integers. Denote (after E. Lehmer and R. K. Guy [1, Problem F6]) by $\Omega(a, b)$ the least number such that for all $p>p(a, b)$ there exists $n \leq \Omega(a, b)$ such that each of $n, n+a, n+b$ is a quadratic residue modulo $p$; moreover, write $\Omega(a, b)=\infty$ if there is no such finite number. There is a conjecture of R. K. Guy and E. Lehmer ([1, Problem F6]) that

if $(a, b) \not \equiv(1,2) \bmod 3,(1,3) \bmod 5,(2,3) \bmod 5,(2,4) \bmod 5,(1,5) \bmod 7$, $(2,3) \bmod 7,(4,6) \bmod 7$, then $\Omega(a, b)<\infty$.

Our modest task at the moment is to formulate this conjecture using explicitly the language of elliptic curves.

Conjecture 1. Consider the elliptic curve $E: Y^{2}=X(X+a)(X+b)$ where $a<b$ are natural numbers. Assume that for each prime $p$ satisfying $p \nmid a b(b-a)$, the duplication does not send all points of $E\left(\mathbb{F}_{p}\right)$ to the point $\infty$ (equivalently $\left|E\left(\mathbb{F}_{p}\right)\right|>4$ ). Then $\Omega(a, b)<\infty$.

The equivalence of both conjectures is easily obtainable by H. Hasse's estimate

$$
|| E\left(\mathbb{F}_{p}\right)|-(p+1)|<2 \sqrt{p},
$$

which implies that $\left|E\left(\mathbb{F}_{p}\right)\right|>4$ for $p \geq 11$. Hence the only candidates for delay primes (using the terminology of E. Lehmer [3]) can be 3,5 or 7 . It is a very elementary verification that $E\left(\mathbb{F}_{p}\right) \cong C_{2} \times C_{2}$ if and only if $(a, b)$ appears in the above list of R. K. Guy and E. Lehmer.

We prove the following result related to Conjecture 1.

THEOREM 3. For any rational numbers $a \neq b$ there exist rational numbers $r_{1}, r_{2}, r_{3}$ satisfying: for every sufficiently large prime number $p$ there exists $j \in\{1,2,3\}$ such that

$$
\left(\frac{r_{j}}{p}\right)=\left(\frac{r_{j}+a}{p}\right)=\left(\frac{r_{j}+b}{p}\right)=+1
$$

where $\left(\bar{p}_{\mathrm{p}}\right)$ stands for the Legendre symbol and the numerators are considered modulo $p$.

For the sake of completeness we formulate a conjecture which is even more basic than Conjecture 1 (see also Problem in [6]). 
Conjecture 2. Consider the elliptic curve $E: Y^{2}=X^{3}+A X^{2}+$ $B X+C$, where $A, B, C$ are given integers. Assume that for each prime number $p$ at which $E$ has good reduction, the reduced curve $E\left(\mathbb{F}_{p}\right)$ contains at least one affine point (equivalently $\left|E\left(\mathbb{F}_{p}\right)\right|>1$ ). Then there exists a constant $C(E)$ such that for each sufficiently large prime $p$ there exist integers $X, Y$ satisfying the congruence

$$
Y^{2} \equiv X^{3}+A X^{2}+B X+C(\bmod p)
$$

and additionally $1 \leq X \leq C(E)$.

For the proofs we need three lemmas.

Lemma 1. If $f(X)=X^{3}+A X+B$ then the following identity holds:

$$
f\left(\frac{\alpha^{3}+4 B}{-4 A-3 \alpha^{2}}\right)=\left(\frac{\alpha^{3}+4 A \alpha-8 B}{-4 A-3 \alpha^{2}}\right)^{2} \cdot \frac{\alpha^{3}+A \alpha+B}{-4 A-3 \alpha^{2}} .
$$

LemMa 2. Let $F$ be a field of characteristic unequal to 2 or 3 and consider the algebraic set $V$ defined over $F$ by the equation

$$
y^{2}+12 A x^{2}=z^{3}+A z+B=: f(z), \quad \text { where } A, B \in F \text { and } A \neq 0 .
$$

Then $V$ is irreducible and if we put

$$
X_{1}=\frac{y z+4 A x}{y-3 x z}, \quad X_{2}=\frac{z^{3}+4 B}{-4 A-3 z^{2}}, \quad X_{3}=\frac{X_{1}^{3}+4 B}{-4 A-3 X_{1}^{2}},
$$

then

$$
\begin{aligned}
& f\left(X_{1}\right) f\left(X_{2}\right) f\left(X_{3}\right) \\
& \quad=\left(\frac{\left(X_{1}^{3}+A X_{1}+B\right)\left(X_{1}^{3}+4 A X_{1}-8 B\right)\left(z^{3}+4 A z-8 B\right)(y-3 x z)}{\left(-4 A-3 X_{1}^{2}\right)\left(-4 A-3 z^{2}\right)^{2}}\right)^{2},
\end{aligned}
$$

where both sides are to be understood as elements of $F(V)$, the function field of $V$.

Proof. For the proof of irreducibility of $V$ we refer to [5, p. 68, Corollary 2 and Corollary 3]. By Lemma 1 we obtain

$$
\begin{aligned}
& f\left(X_{2}\right)=\left(\frac{z^{3}+4 A z-8 B}{-4 A-3 z^{2}}\right)^{2} \cdot \frac{z^{3}+A z+B}{-4 A-3 z^{2}}, \\
& f\left(X_{3}\right)=\left(\frac{X_{1}^{3}+4 A X_{1}-8 B}{-4 A-3 X_{1}^{2}}\right)^{2} \cdot \frac{X_{1}^{3}+A X_{1}+B}{-4 A-3 X_{1}^{2}},
\end{aligned}
$$

and hence

$$
\begin{aligned}
& f\left(X_{1}\right) f\left(X_{2}\right) f(\left.X_{3}\right)=\frac{z^{3}+A z+B}{\left(-4 A-3 X_{1}^{2}\right)\left(-4 A-3 z^{2}\right)} \\
& \quad \times\left(\frac{\left(X_{1}^{3}+A X_{1}+B\right)\left(X_{1}^{3}+4 A X_{1}-8 B\right)\left(z^{3}+4 A z-8 B\right)}{\left(-4 A-3 X_{1}^{2}\right)\left(-4 A-3 z^{2}\right)}\right)^{2} .
\end{aligned}
$$


We use the equation defining $V$ and transform further

$$
\frac{z^{3}+A z+B}{\left(-4 A-3 X_{1}^{2}\right)\left(-4 A-3 z^{2}\right)}=\frac{y^{2}+12 A x^{2}}{\left(-4 A-3 X_{1}^{2}\right)\left(-4 A-3 z^{2}\right)}=\left(\frac{y-3 x z}{-4 A-3 z^{2}}\right)^{2} \text {. }
$$

Now the assertion follows by combining the above formulas.

LEMma 3. Let $V$ be the variety given by (3). Then we have rational morphism

$$
\Psi: F^{2} \rightarrow V(F),
$$

given by the formulas

$$
\Psi(t, s)=(x(t, s), y(t, s), z(t, s)),
$$

where

$$
\begin{aligned}
& x=x(t, s)=3 m^{2} r-4 A m t r+A^{2} t^{2} r-12 A r^{3}-12 A^{2} r s^{2} \\
& +3 B m t s-A B t^{2} s+12 A B s^{3}, \\
& \begin{aligned}
& y=y(t, s)=m^{3}-2 A m^{2} t+A^{2} m t^{2}- 36 A m r^{2}-12 A^{2} m s^{2} \\
&+ B^{2} t^{3}+24 A^{2} t r^{2}-36 A B t r s, \\
& z=z(t, s)=m^{2}+12 A r^{2}, \quad \\
& m=m(t, s):=\frac{A t^{2}-12 A s^{2}}{2 t}, \quad r=r(t, s):=\frac{B t^{2}-1}{24 A s} .
\end{aligned}
\end{aligned}
$$

Proof. This result belongs to Mordell. It is a special case of Theorem 1 on page 113 of his classical treatise [4]. We have only performed all detailed elementary calculations to make the solution as explicit as possible.

Proof of Theorem 1. We apply Lemmas 2 and 3. When we substitute $s=t$ in the formulas for $x, y, z$ in Lemma 3 and then compute $X_{1}, X_{2}, X_{3}$ of Lemma 2 we shall obtain $X_{1}\left(t^{2}\right), X_{2}\left(t^{2}\right), X_{3}\left(t^{2}\right)$, where $X_{1}(t), X_{2}(t), X_{3}(t)$ are displayed in Theorem 1 . Now we will verify that $D_{1}(t) D_{2}(t) D_{3}(t) \neq 0$ and that $X_{1}(t), X_{2}(t), X_{3}(t)$ are non-constant. The lowest term in the product $D_{1}(t) D_{2}(t) D_{3}(t)$ is $144 A^{2} t$ (this follows from the tables given at the end of the paper). Since $A \neq 0$ and $\operatorname{char} F \neq 2,3$ we obtain $144 A^{2} \neq 0$ and hence $D_{1}(t) D_{2}(t) D_{3}(t) \neq 0$.

The equality $N_{1}(t) / D_{1}(t)=c_{1} \in F$ would imply $N_{1}(t)=0$. From Table 1 we see that the coefficients $212 A^{2}, 208 A^{2} B$ and $161568 A^{5}+264 A^{2} B^{2}$ vanish. The fact that $212 A^{2}=0$, while $A$ is not zero, implies that char $F=53$. Since $208 \not \equiv 0(\bmod 53)$, we have $B=0$ and hence, as $161568 \not \equiv 0(\bmod 53)$, we find $A=0$, a contradiction.

Next $X_{2}(t) \notin F$ because $N_{2}(0) \neq 0$ and $D_{2}(0)=0$. For the proof that $X_{3}(t) \notin F$ we use the formula

$$
X_{3}(t)=\frac{X_{1}(t)^{3}+4 B}{-4 A-3 X_{1}(t)^{2}} .
$$


The equality $X_{3}(t)=c_{3} \in F$ would give

$$
X_{1}(t)^{3}+3 c_{3} X_{1}(t)^{2}+\left(4 B+4 c_{3} A\right)=0,
$$

but $c_{3}=X_{3}(0)=-B / A$, which leads to

$$
X_{1}(t)^{2}\left(X_{1}(t)-\frac{3 B}{A}\right)=0,
$$

a contradiction with $X_{1}(t) \notin F$.

Proof of Theorem 2. The set

$$
\left\{t \in T \mid D_{1}\left(t^{2}\right) D_{2}\left(t^{2}\right) D_{3}\left(t^{2}\right)=0\right\}
$$

has no more than $\operatorname{deg} D_{1}(t)+\operatorname{deg} D_{2}(t)+\operatorname{deg} D_{3}(t) \leq 25$ elements. For the remaining values of $t$ the map $\Phi$ is well defined because of (1) and the fact that $\mathbb{F}_{q}^{*}$ is cyclic. In the worst case the same point in $E\left(\mathbb{F}_{q}\right)$ can be obtained by our procedure for $\operatorname{deg} X_{1}(t)+\operatorname{deg} X_{2}(t)+\operatorname{deg} X_{3}(t) \leq 26$ values of $t \in T$, and this ends the proof of inequality (2).

Proof of Corollary 1. Obviously we can assume that $|F|>49$. First, take a subset $T$ of $F$ satisfying $T \cap(-T)=\emptyset$ and $|T|=26$. Secondly, using Theorem 2 find $X \in F$ such that $f(X) \in F^{2}$. Finally, compute the relevant square root $Y=\sqrt{f(X)}$ using $n \in F \backslash F^{2}$ and the well known "approximation" procedure due to D. Shanks [8], in time polynomial in $\log |F|$.

First proof of Corollary 2. We distinguish two cases. If $f(0)=B$ is a quadratic residue $\bmod p$ we compute $y=\sqrt{B} \bmod p$, using the algorithm of R. Schoof [7], in time $O\left(\left(|B|^{1 / 2+\varepsilon} \log p\right)^{9}\right)$. If $B$ is a quadratic non-residue we put $n=B$ and apply Corollary 1 .

Second (direct) proof of Corollary 2. The proof differs from the above only in the case $\left(\frac{B}{p}\right)=-1$. So we can compute all square roots which appear in what follows in deterministic polynomial time. By Lemma 1 it is sufficient to find $\alpha \in \mathbb{Z}$ satisfying

$$
\left(\frac{-4 A-3 \alpha^{2}}{p}\right)=-1
$$

Consider the quadratic form

$$
g(u, v)=-4 A u^{2}-3 v^{2} .
$$

If we find $u, v \in \mathbb{F}_{p}$ such that

$$
\left(\frac{g(u, v)}{p}\right)=-1 \quad \text { and } \quad u v \neq 0
$$

then we put $\alpha=v / u$ and (4) holds. If either $-4 A=g(1,0)$ or $-3=g(0,1)$ is a quadratic non-residue mod $p$ then we easily find $u, v \in \mathbb{F}_{p}^{*}$ satisfying $g(u, v)=-4 A$ or $g(u, v)=-3$ (respectively) and (5) holds. If both $-4 A$ 
and -3 are squares mod $p$ we first find $c, d \in \mathbb{F}_{p}^{*}$ such that

$$
c^{2}=-4 A, \quad d^{2}=-3 .
$$

In new variables $s=c u, t=d v$ the form $g(u, v)$ transforms to $G(s, t)=$ $s^{2}+t^{2}$. Let $n_{0}$ be the unique number in the interval [2,p-1] satisfying $n_{0} \equiv B \bmod p$ and define

$$
n_{j+1}=n_{j}-\left[\sqrt{n_{j}}\right]^{2} \quad \text { for } j=0,1, \ldots
$$

For $J=\max \left\{j \geq 0 \mid\left(\frac{n_{j}}{p}\right)=-1\right\}$ we obtain

$$
n_{J}=G\left(f,\left[\sqrt{n_{J}}\right]\right)
$$

with $f \in \mathbb{F}_{p}^{*}$ satisfying $f^{2}=n_{J+1}$. Since $J \ll \log \log p$ we have arrived at $u=f / c, v=\left[\sqrt{n_{J}}\right] / d$ satisfying (5) in polynomial time.

Proof of Theorem 3. Consider the elliptic curve

$$
\widetilde{E}: \widetilde{Y}^{2}=\widetilde{X}(\widetilde{X}+a)(\widetilde{X}+b)=: g(\widetilde{X}) .
$$

The map

$$
\widetilde{X} \mapsto X=\widetilde{X}+\frac{a+b}{3}, \quad \widetilde{Y} \mapsto Y
$$

gives an isomorphism of $\widetilde{E}$ onto $E: Y^{2}=X^{3}+A X+B$, where $A=$ $\left(a b-a^{2}-b^{2}\right) / 3 \neq 0$. We apply Theorem 1 to obtain rational numbers $\widetilde{X}_{1}, \widetilde{X}_{2}, \widetilde{X}_{3}, \widetilde{U}$ such that

$$
g\left(\widetilde{X}_{1}\right) g\left(\widetilde{X}_{2}\right) g\left(\widetilde{X}_{3}\right)=\widetilde{U}^{2} \text { and } \widetilde{U} \neq 0 .
$$

Now we define

$$
r_{j}=\frac{\left(\widetilde{X}_{j}-a b\right)^{2}}{4 g\left(\widetilde{X}_{j}\right)}
$$

and verify that

$$
r_{j}+a=\frac{\left(\widetilde{X}_{j}^{2}+2 a \widetilde{X}_{j}+a b\right)^{2}}{4 g\left(\widetilde{X}_{j}\right)}, \quad r_{j}+b=\frac{\left(\widetilde{X}_{j}^{2}+2 b \widetilde{X}_{j}+a b\right)^{2}}{4 g\left(\widetilde{X}_{j}\right)} .
$$

If a prime number $p$ appears neither in the numerators nor in the denominators of $g\left(\widetilde{X}_{j}\right)(j=1,2,3)$, then using the fact that $\mathbb{F}_{p}^{*}$ is cyclic and (6) we infer that at least one of $g\left(\widetilde{X}_{j}\right)$ is a square in $\mathbb{F}_{p}$. The desired assertion follows now by the definition of $r_{j}$ and (7).

Table $1(i=1)$

$$
\begin{gathered}
n_{0,0}^{(1)}=212 ; \quad n_{0,1}^{(1)}=-208 ; \quad n_{3,0}^{(1)}=-161568, \quad n_{0,2}^{(1)}=-264 ; \\
n_{3,1}^{(1)}=441408, \quad n_{0,3}^{(1)}=304 ; \quad n_{6,0}^{(1)}=-92765376, \quad n_{3,2}^{(1)}=-127776, \quad n_{0,4}^{(1)}=-44 ; \\
d_{0,0}^{(1)}=-1 ; \quad d_{0,1}^{(1)}=5 ; \quad d_{3,0}^{(1)}=10536, \quad d_{0,2}^{(1)}=-10 ; \quad d_{3,1}^{(1)}=9480, \quad d_{0,3}^{(1)}=10 ; \\
d_{6,0}^{(1)}=4024944, \quad d_{3,2}^{(1)}=-4488, \quad d_{0,4}^{(1)}=-5 ; \quad d_{6,1}^{(1)}=2108304, \quad d_{3,3}^{(1)}=2904, \quad d_{0,5}^{(1)}=1 .
\end{gathered}
$$


Table $2(i=2)$

$n_{0,0}^{(2)}=-1 ; \quad n_{0,1}^{(2)}=6 ; \quad n_{3,0}^{(2)}=-4356, \quad n_{0,2}^{(2)}=-15 ; \quad n_{3,1}^{(2)}=-424944, n_{0,3}^{(2)}=20 ;$ $n_{6,0}^{(2)}=-6324912, n_{3,2}^{(2)}=-26136, n_{0,4}^{(2)}=-15 ;$ $n_{6,1}^{(2)}=12649824, n_{3,3}^{(2)}=17424, n_{0,5}^{(2)}=6 ;$ $n_{9,0}^{(2)}=-3061257408, n_{6,2}^{(2)}=-6324912, n_{3,4}^{(2)}=-4356, n_{0,6}^{(2)}=-1 ;$ $d_{0,0}^{(2)}=1 ; \quad d_{0,1}^{(2)}=-4 ; \quad d_{3,0}^{(2)}=5976, \quad d_{0,2}^{(2)}=6 ; \quad d_{3,1}^{(2)}=-5808, \quad d_{0,3}^{(2)}=-4 ;$ $d_{6,0}^{(2)}=2108304, d_{3,2}^{(2)}=2904, d_{0,4}^{(2)}=1$.

Table $3(i=3,31,32)$

$n_{0,1}^{(3)}=1 ; \quad n_{3,0}^{(3)}=0, n_{0,2}^{(3)}=-15 ; \quad n_{3,1}^{(3)}=-31608, n_{0,3}^{(3)}=105$ $n_{6,0}^{(3)}=-2382032, n_{3,2}^{(3)}=287640, n_{0,4}^{(3)}=-455$; $n_{6,1}^{(3)}=327958320, n_{3,3}^{(3)}=-1124496, n_{0,5}^{(3)}=1365 ;$ $n_{9,0}^{(3)}=5446134144, n_{6,2}^{(3)}=-949378416, n_{3,4}^{(3)}=2369808, n_{0,6}^{(3)}=-3003 ;$ $n_{9,1}^{(3)}=-940697745408, n_{6,3}^{(3)}=-185899568, n_{3,5}^{(3)}=-2531880, n_{0,7}^{(3)}=5005$; $n_{12,0}^{(3)}=-1023635467008, n_{9,2}^{(3)}=-4041852271488$, $n_{6,4}^{(3)}=3844905120, n_{3,6}^{(3)}=-14904, n_{0,8}^{(3)}=-6435$; $n_{12,1}^{(3)}=-1271178606627072, n_{9,3}^{(3)}=-557953136640$, $n_{6,5}^{(3)}=-5637798432, n_{3,7}^{(3)}=4402080, n_{0,9}^{(3)}=6435 ;$ $n_{15,0}^{(3)}=-3711755775062016, n_{12,2}^{(3)}=-3365703371771136$, $n_{9,4}^{(3)}=1809225932544, n_{6,6}^{(3)}=2558454048, n_{3,8}^{(3)}=-7401888, n_{0,10}^{(3)}=-5005 ;$ $n_{15,1}^{(3)}=-502999567986972672, n_{12,3}^{(3)}=-924766944152832$, $n_{9,5}^{(3)}=-3401013749760, n_{6,7}^{(3)}=1784103840, n_{3,9}^{(3)}=7013304, n_{0,11}^{(3)}=3003 ;$ $n_{18,0}^{(3)}=447914759358173184, n_{15,2}^{(3)}=-981669643253544960, n_{12,4}^{(3)}=329477012308224$, $n_{9,6}^{(3)}=913161021696, n_{6,8}^{(3)}=-3372070032, n_{3,10}^{(3)}=-4408920, n_{0,12}^{(3)}=-1365 ;$

$n_{18,1}^{(3)}=-73786028437373497344, n_{15,3}^{(3)}=-459570852044992512$, $n_{12,5}^{(3)}=-977913669655296, n_{9,7}^{(3)}=439245379584$, $n_{6,9}^{(3)}=2438317040, n_{3,11}^{(3)}=1904112, n_{0,13}^{(3)}=455 ;$

$n_{21,0}^{(3)}=1042769766152244658176, n_{18,2}^{(3)}=-84332284536876355584$, $n_{15,4}^{(3)}=5961076345331712, n_{12,6}^{(3)}=-43484326592256$, $n_{9,8}^{(3)}=-707241693312, n_{6,10}^{(3)}=-1030036656, n_{3,12}^{(3)}=-555120, n_{0,14}^{(3)}=-105$; $n_{21,1}^{(3)}=-2848874263082603053056, n_{18,3}^{(3)}=-63482146340076490752$, $n_{15,5}^{(3)}=-101522561076541440, n_{12,7}^{(3)}=69490543161600$, $n_{9,9}^{(3)}=280657428480, n_{6,11}^{(3)}=255430032, n_{3,13}^{(3)}=100584, n_{0,15}^{(3)}=15$; 


$$
\begin{aligned}
& n_{24,0}^{(3)}=199571139166470771769344, n_{21,2}^{(3)}=824674128787069304832, \\
& n_{18,4}^{(3)}=-7951403445605351424, n_{15,6}^{(3)}=-37420516674680832, n_{12,8}^{(3)}=-66000709716480 \text {, } \\
& n_{9,10}^{(3)}=-61039617408, n_{6,12}^{(3)}=-31603264, n_{3,14}^{(3)}=-8712, n_{0,16}^{(3)}=-1 ; \\
& d_{0,0}^{(31)}=-1 ; \quad d_{0,1}^{(31)}=5 ; \quad d_{3,0}^{(31)}=10536, \quad d_{0,2}^{(31)}=-10 ; \quad d_{3,1}^{(31)}=9480, \quad d_{0,3}^{(31)}=10 ; \\
& d_{6,0}^{(31)}=4024944, d_{3,2}^{(31)}=-4488, d_{0,4}^{(31)}=-5 ; \\
& d_{6,1}^{(31)}=2108304, d_{3,3}^{(31)}=2904, d_{0,5}^{(31)}=1 ; \\
& d_{0,0}^{(32)}=1 ; \quad d_{0,1}^{(32)}=-10 ; \quad d_{3,0}^{(32)}=12636, \quad d_{0,2}^{(32)}=45 ; \quad d_{3,1}^{(32)}=20256, \quad d_{0,3}^{(32)}=-120 ; \\
& d_{6,0}^{(32)}=51578784, d_{3,2}^{(32)}=-158448, d_{0,4}^{(32)}=210 ; \\
& d_{6,1}^{(32)}=426572352, d_{3,3}^{(32)}=149472, d_{0,5}^{(32)}=-252 ; \\
& d_{9,0}^{(32)}=74892394368, d_{6,2}^{(32)}=-178487712, d_{3,4}^{(32)}=146472, d_{0,6}^{(32)}=210 ; \\
& d_{9,1}^{(32)}=42705805824, d_{6,3}^{(32)}=-194173056, d_{3,5}^{(32)}=-328224, d_{0,7}^{(32)}=-120 ; \\
& d_{12,0}^{(32)}=38682048607488, d_{9,2}^{(32)}=217678171392, \\
& d_{6,4}^{(32)}=339663456, d_{3,6}^{(32)}=208656, d_{0,8}^{(32)}=45 ; \\
& d_{12,1}^{(32)}=-44449457564160, d_{9,3}^{(32)}=-122450296320, \\
& d_{6,5}^{(32)}=-126498240, d_{3,7}^{(32)}=-58080, d_{0,9}^{(32)}=10 ; \\
& d_{15,0}^{(32)}=6454061238316032, d_{12,2}^{(32)}=22224728782080, \\
& d_{9,4}^{(32)}=30612574080, d_{6,6}^{(32)}=21083040, d_{3,8}^{(32)}=7260, d_{0,10}^{(32)}=1 .
\end{aligned}
$$

\section{References}

[1] R. K. Guy, Unsolved Problems in Number Theory, 2nd ed., Springer, 1994.

[2] N. Koblitz, Algebraic Aspects of Cryptography, Springer, Berlin, 1998.

[3] E. Lehmer, Patterns of power residues, J. Number Theory 17 (1983), 37-46.

[4] L. J. Mordell, Diophantine Equations, Academic Press, London and New York, 1969.

[5] A. Schinzel, Polynomials with Special Regard to Reducibility, Cambridge Univ. Press, 2000.

[6] A. Schinzel and M. Skałba, On equations $y^{2}=x^{n}+k$ in a finite field, Bull. Polish Acad. Sci. Math. 52 (2004), 223-226.

[7] R. Schoof, Elliptic curves over finite fields and the computation of square roots mod $p$, Math. Comp. 44 (1985), 483-494.

[8] D. Shanks, Five number-theoretic algorithms, Congr. Numer. 7 (1972), 51-70.

Institute of Mathematics

Polish Academy of Sciences

00-956 Warszawa, Poland

E-mail: skalba@impan.gov.pl
Institute of Mathematics University of Warsaw Banacha 2 02-097 Warszawa, Poland

Received on 1.10.2004

and in revised form on 18.1.2005 